\title{
Transesophageal Bronchoscopic Ultrasound-Guided Fine Needle Aspiration for Diagnosis of Sarcoidosis
}

\author{
Masahide Oki ${ }^{a}$ Hideo Saka ${ }^{a}$ Chiyoe Kitagawa ${ }^{a}$ Yoshihito Kogure ${ }^{a}$ \\ Naohiko Murata ${ }^{a}$ Takashi Adachi $^{a}$ Shu Ichihara ${ }^{b}$ Suzuko Moritani ${ }^{b}$ \\ ${ }^{a}$ Department of Respiratory Medicine and b Department of Pathology, Nagoya Medical Center, Nagoya, Japan
}

\section{Key Words}

Endobronchial ultrasound - Endoscopic ultrasound •

Sarcoidosis · Transbronchial needle aspiration - Endoscopic ultrasound with bronchoscope-guided fine needle aspiration · Bronchoscopy

\section{Abstract \\ Background: Several studies have reported that specimens from mediastinal lesions located adjacent to the esophagus can be sampled using an ultrasound bronchoscope instead of an ultrasound endoscope. Objectives: The aim of this study was to evaluate the diagnostic utility of transesoph- ageal bronchoscopic ultrasound-guided fine needle aspi- ration using an ultrasound bronchoscope in patients with stage I/II sarcoidosis. Methods: Thirty-three patients sus- pected of having stage $\mathrm{I} / \mathrm{II}$ sarcoidosis were included in this prospective study. Needle aspiration through the esopha- gus using an ultrasound bronchoscope was performed for hilar and/or mediastinal lymph nodes. The final diagnosis of sarcoidosis was based on clinicoradiological compatibility and pathological findings. Results: A total of 62 lymph nodes with a mean shortest diameter of $13.6 \mathrm{~mm}$ were examined. Of the 33 patients enrolled, 29 were given a final diagnosis of sarcoidosis. Four of the residual patients had other dis-}

eases (1 lung cancer, 1 tuberculosis, 2 non-specific lymphadenitis). Transesophageal bronchoscopic ultrasound-guided fine needle aspiration showed noncaseating epithelioid cell granulomas in 25 of 29 patients $(86 \% ; 95 \%$ confidence interval 73-100) with the final diagnosis of sarcoidosis. No complications were observed. Conclusions: Transesophageal bronchoscopic ultrasound-guided fine needle aspiration is feasible, safe and accurate for the diagnosis of stage I/II sarcoidosis.

Copyright $\odot 2012$ S. Karger AG, Basel

\section{Introduction}

Sarcoidosis is a multisystem granulomatous disease of unknown etiology. We commonly encounter patients with the disease in our daily clinical practice. Sarcoidosis often presents characteristic clinical and radiological pictures, and thus, the clinical diagnosis is highly reliable.

Preliminary data were previously presented at the CHEST 2011 annual meeting (trial registration: UMIN-Clinical Trials Registry; identifier: UMIN0000002883; http://www.umin.ac.jp/ctr/index.htm).

\section{KARGER \\ Fax +41613061234 \\ E-Mail karger@karger.ch}

www.karger.com
(C) 2012 S. Karger AG, Basel

0025-7931/13/0852-0137\$38.00/0

Accessible online at:

www.karger.com/res
Masahide Oki

Department of Respiratory Medicine, Nagoya Medical Center

4-1-1 Sannomaru, Naka-ku

Nagoya 460-0001 (Japan)

E-Mail masahideo@aol.com 
However, pathological confirmation demonstrating noncaseating epitheliod cell granulomas is essential for its definitive diagnosis [1]. So far, a transbronchial approach using a flexible bronchoscope including transbronchial lung biopsy (TBLB) and endobronchial biopsy has been the most common method for sampling specimens in patients with suspected sarcoidosis [1]. However, the yield of these conventional methods for detecting noncaseating epithelioid cell granulomas is not satisfactory, especially for stage I sarcoidosis [2-5]. In addition, it is well known that TBLB sometimes causes pneumothorax and/ or bleeding. Since the advent of ultrasound endoscopes which make it possible to perform transesophageal endoscopic ultrasound-guided fine needle aspiration (EUSFNA) and ultrasound bronchoscopes enabling endobronchial ultrasound-guided transbronchial needle aspiration (EBUS-TBNA), many investigators have reported the usefulness of these procedures for diagnosing benign as well as malignant hilar/mediastinal lesions $[6,7]$. The reported diagnostic yields of EUS-FNA and EBUS-TBNA for sarcoidosis demonstrating epithelioid cell granulomas are excellent, ranging from 82 to 100 [8-12] and from 71 to $94 \%[2,4,5,12-16]$, respectively. Furthermore, these procedures rarely cause complications.

Recently, several investigators [17-22] suggested that needle aspiration procedure through the esophagus can be performed using an US bronchoscope instead of an US endoscope. The procedure may be preferable to EUSFNA for some pulmonologists because it obviates the need for an ultrasound endoscope and an experienced endoscopist. The aim of this study was to evaluate the diagnostic accuracy, feasibility and safety of transesophageal endoscopic ultrasound with bronchoscope-guided fine needle aspiration (EUS-B-FNA) in patients with stage I/II sarcoidosis.

\section{Patients and Methods}

\section{Patients}

We carried out a prospective study which was approved by the institutional review board of Nagoya Medical Center (identifier 2009-254) and registered with the University Hospital Medical Information Network-Clinical Trials Registry (identifier UMIN000002883). From January 2010 until February 2011, a total of 33 patients with suspected stage I (bilateral hilar and/or mediastinal lymphadenopathy) or stage II (bilateral hilar and/or mediastinal lymphadenopathy and parenchymal abnormalities) sarcoidosis in the presence of a compatible clinical and radiological picture were enrolled in the study. Patients with already pathologically diagnosed sarcoidosis were excluded. All patients gave written informed consent for their participation.
The primary outcome of this study was to evaluate the pathological diagnostic yield of EUS-B-FNA for stage I and II sarcoidosis. The secondary outcome was to assess the safety and feasibility of this procedure.

\section{Procedures}

EUS-B-FNA was performed using a $7.5-\mathrm{MHz}$ curvilinear scanning ultrasound bronchoscope (BF-UC260F-OL8; Olympus, Tokyo, Japan) connected to an ultrasound processor (EU-C2000; Olympus). The procedure was performed at the left lateral position, which is similar to that during esophagogastroduodenoscopy including EUS-FNA to prevent aspiration, under conscious sedation using midazolam by staff pulmonologists. After anesthetizing the upper airway with lidocaine, an ultrasound bronchoscope was inserted and advanced through the esophagus while examining the structure around the esophagus by ultrasound. Once the target lesion was identified, it was punctured through the esophagus with a 21-gauge needle under real-time ultrasound guidance. After that, the needle was manipulated back and forward within the lesion, applying suction by confirming the ultrasound image, and then retracted to collect the aspirated specimens.

Handling of sampled specimens was done in the same manner as in the EBUS-TBNA procedures we previously described [23]. The aspirated specimen in the needle was pushed out by a stylet and then expelled by blowing air through a syringe onto a glass slide. The visible tissue fragments on the glass slide were then collected and transferred into containers filled with formalin for histologic examination, and the remaining specimen on the glass slide was immediately smeared and fixed in 95\% alcohol for cytologic examination. The remaining specimen stored at the lumen of the needle and catheter was then washed and flushed into saline for culture for microbiological analysis. The pathological specimens obtained by EUS-B-FNA in the containers labeled with the lymph node station were then submitted to the pathologist for interpretation. On-site cytologic evaluation was not used. Decision on the number of punctures or target lesions depended on the examiner. If oxygen saturation decreased to less than $90 \%$ for more than 20 s during the procedure, oxygen supplementation was provided to maintain oxygen saturation at more than $90 \%$. The lymph node location examined [24], the number of punctures, supplemental oxygen administration and the time of the procedure measured were recorded.

\section{Diagnosis}

Each histologic and cytologic specimen was interpreted separately as 'positive', 'suspicious' or 'negative' for sarcoidosis with noncaseating epithelioid cell granulomas by an experienced pathologist. 'Suspicious' findings were analyzed as negative in this study. The final diagnosis of sarcoidosis was based on the clinical and radiological compatibility at the time of the procedures and during the clinical follow-up period as well as on the pathological findings of noncaseating granulomas and on exclusion of other causes of granulomas.

\section{Data Analysis}

EUS-B-FNA may be promising in terms of less invasiveness compared to TBLB, which is the standard method, because the procedure is rarely associated with complications including bleeding and pneumothorax. We estimated that 30 patients would 
be required, calculated under the following conditions: alternative diagnostic yield 85\% [8-12], null diagnostic yield 65\% [25] and a statistical power of $90 \%$ at a one-sided significance level of 0.1 . We arranged to enroll 33 patients to compensate for a dropout rate of $10 \%$.

Statistical analyses were performed using a statistical software program (PASW Statistics 18; SPSS Inc., Chicago, Ill., USA). Means and percentages were presented as appropriate.

\section{Results}

\section{Patients and Procedures}

Characteristics of patients and lesions are shown in table 1. Among the 33 patients enrolled in this study, 18 patients had suspected stage I and 15 patients suspected stage II sarcoidosis. EUS-B-FNA was performed for 62 lymph nodes (mean shortest diameter on chest computed tomography $13.6 \mathrm{~mm}$ ) with a mean of 3.3 punctures per lesion in 32 patients (97\%). EUS-B-FNA was not performed in the remaining patient because a puncturable lymph node could not be detected by ultrasound. Temporary supplemental oxygen was administered in 2 patients (6\%). All patients tolerated the procedure well and no events such as cough, vomiting or dyspnea prevented continuation of the procedure. The median procedure time was $22.5 \mathrm{~min}$ (range 12.8-40.5).

A diagnostic flow chart is shown in figure 1. EUS-BFNA showed noncaseating epithelioid cell granulomas in 25 patients, and all patients were judged to have sarcoidosis from the clinicoradiological compatibility (fig. 2). Three patients without definitive pathological findings by EUS-B-FNA underwent EBUS-TBNA afterwards. EBUS-TBNA provided a positive result in 2 patients and a suspicious result in 1 patient. One patient with suspicious pathological findings for sarcoidosis by EUS-BFNA with clinicoradiological compatibility was judged to have sarcoidosis. The remaining 4 patients had other diseases - tuberculosis in 1 (fig. 3), lung cancer in 1 and nonspecific lymphadenitis in 2 subjects. Thus, a total of 29 patients were given a final diagnosis of sarcoidosis. Overall, EUS-B-FNA provided diagnostic materials in 27 of 33 patients (82\%).

\section{Diagnostic Yield in Patients with Sarcoidosis}

EUS-B-FNA confirmed a diagnosis of sarcoidosis by providing specimens with noncaseating epithelioid cell granulomas in 14 of 17 patients (82\%) with stage I sarcoidosis, in 11 of 12 patients (92\%) with stage II sarcoidosis and overall in 25 of 29 patients (86\%; $95 \%$ confidence interval 73-100). There was no significant difference in the
Table 1. Characteristics of patients and lesions

\begin{tabular}{|c|c|}
\hline Characteristics & Data \\
\hline Patients & 33 \\
\hline Mean age $\pm S D$, years & $51.5 \pm 18.5(25-82)$ \\
\hline Males/females & $18 / 15$ \\
\hline \multicolumn{2}{|l|}{ Chest radiographic staging } \\
\hline Stage I/II & $18 / 15$ \\
\hline \multicolumn{2}{|l|}{ Serum angiotensin-converting enzyme } \\
\hline$>21.4 / \leq 21.4 \mathrm{U} / 1$ & $8 / 25$ \\
\hline \multicolumn{2}{|l|}{ Bronchoscopy } \\
\hline $\begin{array}{l}\text { Initial bronchoscopy/previous } \\
\text { non-diagnostic bronchoscopy }\end{array}$ & $29 / 4$ \\
\hline Total LN number evaluated by EUS-B-FNA & 62 \\
\hline Shortest LN diameter, $\mathrm{mm}$ & $13.6(6.8-28.7)$ \\
\hline Mean number of punctures per lesion & $3.3(1-7)$ \\
\hline \multicolumn{2}{|l|}{ LN location for EUS-B-FNA } \\
\hline $2 \mathrm{~L}$ & 1 \\
\hline $3 p$ & 1 \\
\hline $4 \mathrm{~L}$ & 22 \\
\hline $4 \mathrm{R}$ & 1 \\
\hline 7 & 30 \\
\hline 8 & 3 \\
\hline $10 \mathrm{~L}$ & 4 \\
\hline Median follow-up duration, months & $17(1-26)$ \\
\hline
\end{tabular}

Figures in parentheses are ranges. $\mathrm{LN}=$ Lymph node.

diagnostic yield of EUS-B-FNA between stage I and stage II sarcoidosis $(\mathrm{p}=0.44)$. The sensitivity, negative predictive value and accuracy of EUS-B-FNA for pathological diagnosis of sarcoidosis was 86,50 and $88 \%$, respectively. Cytologic and histologic specimens contained diagnostic material in 23 of 29 patients (79\%) and in 24 of 29 patients (83\%), respectively. Among 55 nodal lesions examined in the 29 patients with a final diagnosis of sarcoidosis, noncaseating epithelioid cell granulomas were demonstrated in 44 lesions (80\%) by EUS-B-FNA.

No complications were associated with EUS-B-FNA.

\section{Discussion}

Our study demonstrated a high diagnostic yield (86\%) of EUS-B-FNA demonstrating noncaseating epithelioid granulomas in patients with suspected stage I/II sarcoidosis. In addition, mediastinal observation through the esophagus using an ultrasound bronchoscope could be performed in all 33 patients and specimens could be sampled by pulmonologists in 32 patients without any complications. To our knowledge, this is the first study 
Fig. 1. Diagnostic flow chart. LNs = Lymph

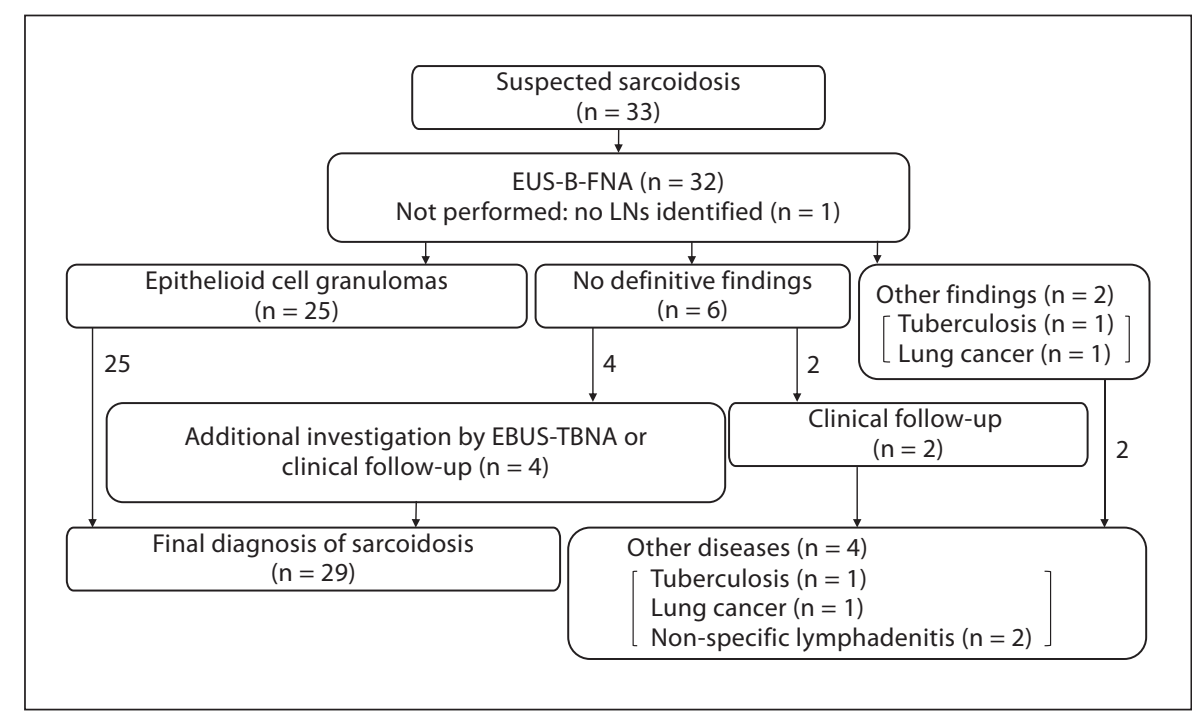

to evaluate the utility of EUS-B-FNA for the pathological diagnosis of sarcoidosis.

For this kind of diagnosis, the specimens must be obtained from the most accessible organ by means of the least invasive procedure. Because the lungs are involved in most cases, the most common procedure so far has been TBLB through a flexible bronchoscope, which is a widespread procedure for sampling specimens from the lungs. However, the diagnostic yield of TBLB, which was reportedly $58 \%$ for stage I and $75 \%$ for stage II sarcoidosis [25], is not satisfactorily high. Additionally, the procedure is occasionally associated with pneumothorax (incidence of 1-5\%) and bleeding (incidence of 9\%) [25]. EUS-FNA and EBUS-TBNA, which are newer methods for diagnosing sarcoidosis, surpass TBLB in accuracy and safety $[2$, $4,5]$. Although sarcoidosis is a benign disease, cytologic diagnosis is highly reliable [26]. Thus, needle aspiration procedures, which can sample both histologic and cytologic specimens, seem to be favorable. Although our study was not designed to be compared with TBLB directly, the diagnostic yield of EUS-B-FNA ( $86 \%$; $95 \%$ confidence interval 73-100) in detection of noncaseating epithelioid cell granulomas seems to be higher than that of TBLB. Furthermore, EUS-B-FNA seems advantageous in terms of less invasiveness.

EUS-FNA introduced in the early 1990s [27] has made it possible to explore lesions around the esophagus. The procedure has been reported to be a safe and accurate method for the diagnosis of hilar/mediastinal benign as well as malignant lesions [6]. Although EUS-FNA is undoubtedly useful, it has some limitations in that it re- quires a skilled endoscopist and dedicated equipment including an ultrasound endoscope and needles. Ultrasound bronchoscopes have a mechanism similar to ultrasound endoscopes. Thus, the needle aspiration procedure through the esophagus can be performed with ultrasound bronchoscopes as well as with ultrasound endoscopes. EUS-B-FNA overcomes the limitations of EUSFNA which require a skilled endoscopist and an ultrasound endoscope; several investigators have demonstrated the feasibility, safety and effectiveness of the procedure for diagnosing benign [21] and malignant diseases [17-20, 22]. Hwangbo et al. [19] reported the effectiveness of adding EUS-B-FNA to EBUS-TBNA in the mediastinal staging of lung cancer. The sensitivity, negative predictive value and accuracy increased from 84 to 91, 93 to 96 and 95 to $97 \%$ by adding EUS-B-FNA to EBUS-TBNA, respectively. Furthermore, no complication associated with EUS-B-FNA was observed in their study. Herth et al. [20] also demonstrated that the combination of EUS-B-FNA and EBUS-TBNA increased the diagnostic accuracy compared with each method alone, without any complications in the mediastinal staging of lung cancer.

EUS-FNA has been reported useful in the diagnosis of sarcoidosis. The reported diagnostic yield for sarcoidosis by detecting noncaseating epithelioid cell granulomas ranged from 82 to $100 \%$ [8-12]. The diagnostic yield of $86 \%$ with EUS-B-FNA in our study seems comparable to that of EUS-FNA. EUS-B-FNA may have another advantage over EUS-FNA in that the primary physician, the pulmonologist (but not the gastroenterologist), who 

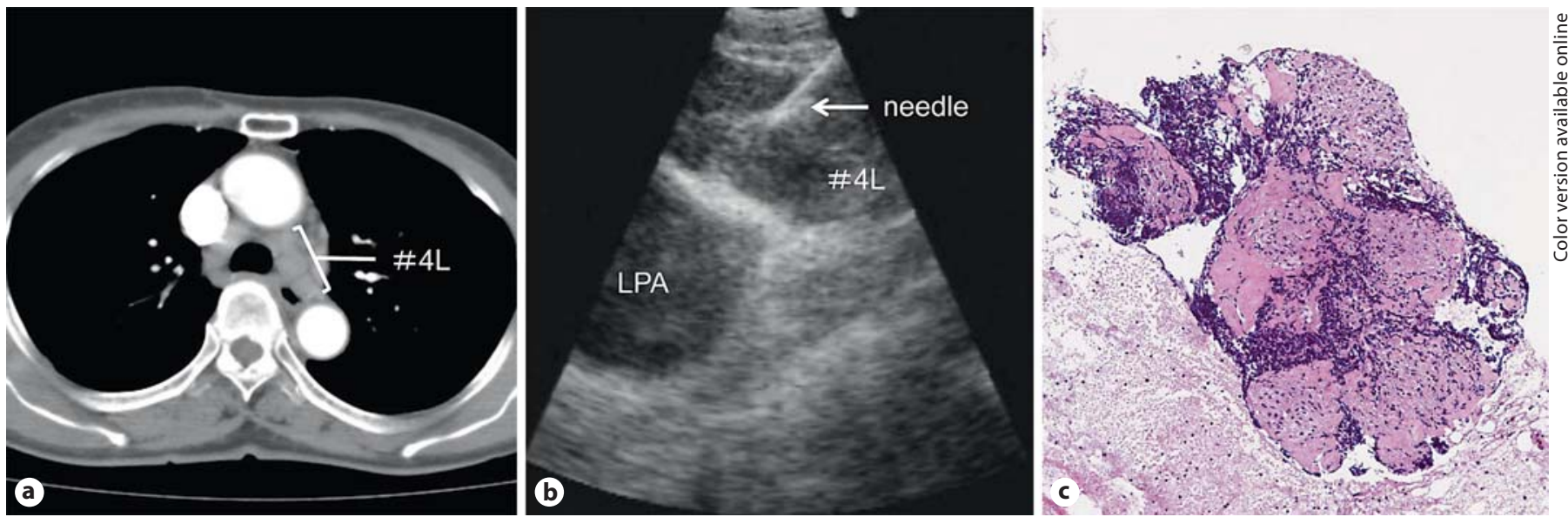

Fig. 2. Transesophageal EUS-B-FNA for the left lower paratracheal lymph node. a Enhanced computed tomographic image. b Ultrasound image. c Specimen obtained by EUS-B-FNA from the left lower paratracheal lymph node $(\# 4 \mathrm{~L})$ containing noncaseating epithelioid cell granulomas suggestive of sarcoidosis. Hematoxylin and eosin. $\times 100 . \mathrm{LPA}=$ Left pulmonary artery.
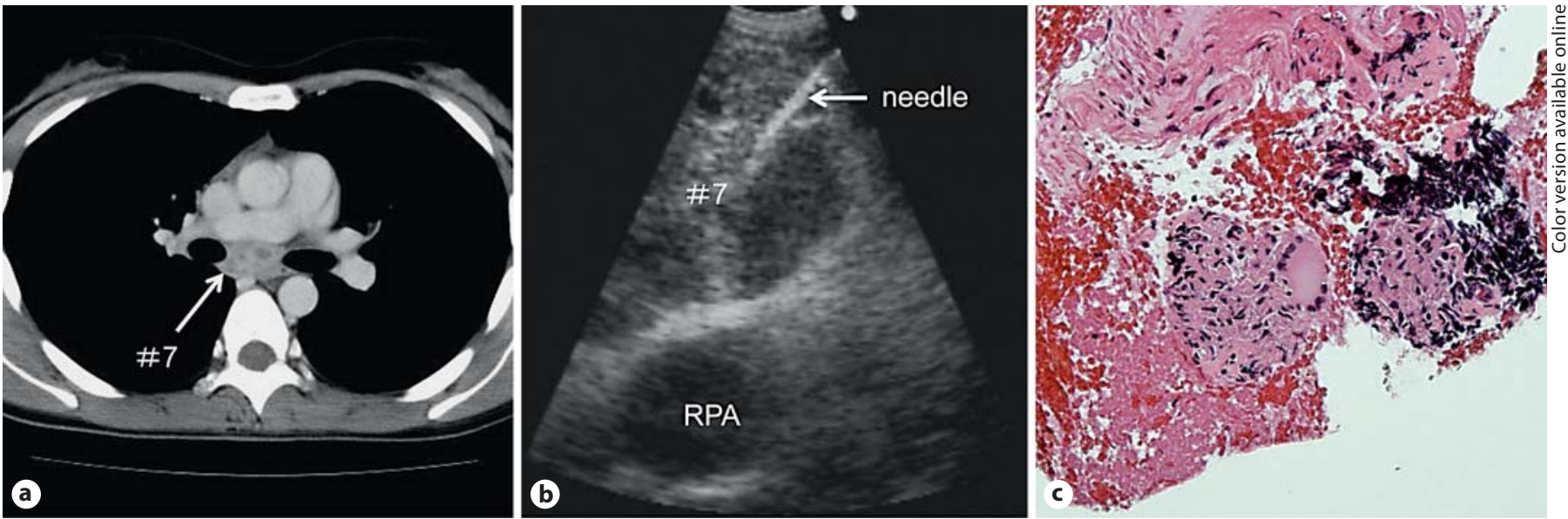

Fig. 3. Transesophageal EUS-B-FNA for subcarinal lymph node. a Enhanced computed tomographic image. b Ultrasound image. c Specimen obtained by EUS-B-FNA for subcarinal lymph node (\#7) showing necrotizing epithelioid cell granulomas with a Langhans-type giant cell suggestive of tuberculosis. Hematoxylin and eosin. $\times 200$. RPA $=$ Right pulmonary artery.

makes the management decision for patients with sarcoidosis as well as many other diseases involving mediastinal lymph nodes, may be able to perform the procedure [28]. In addition, the procedure using an ultrasound bronchoscope, which has only half the outer diameter of an ultrasound endoscope, is likely more tolerable for patients. This procedure cannot replace EUSFNA completely because ultrasound endoscopes have distinct advantages over ultrasound bronchoscopes, such as a larger working channel which enables the use of needles of various size and length, a wider ultrasonic scanning range, as well as better visibility and adjustability of the protruding needle angle, all of which may improve the diagnostic accuracy. However, EUS-BFNA, which provided satisfactory results in our study with a simple procedure that can be performed by pulmonologists for diagnosing sarcoidosis, seems particularly promising. 
EBUS-TBNA is another useful method for the diagnosis of sarcoidosis $[2,4,5,12-16,29]$. The yield of the procedure for the detection of noncaseating epithelioid cell granulomas of sarcoidosis has been reported to range from 71 to $94 \%$ [2, 4, 5, 12-16], and several researchers have indicated that the diagnostic yield of EBUS-TBNA was higher than the yield of conventional methods such as TBLB $[2,4,5]$ and TBNA without ultrasound guidance [16]. The advantage of EBUS-TBNA over EUS-B-FNA or EUS-FNA in the diagnosis of sarcoidois is the higher accessibility to the mediastinal and hilar lymph nodes [19]. Lymph nodes that are located at the right paratracheal and hilar lesions, which are commonly involved with sarcoidosis and difficult to be biopsied by the esophageal approach, can be readily sampled by EBUS-TBNA. However, most patients with stage I/II sarcoidosis have multiple lymph node involvement, including subcarinal lymph nodes and left paratracheal lymph nodes which can be sampled easily with the transesophageal approach. EUS-B-FNA may have the advantage of greater tolerability for patients. Some specialists $[30,31]$ have insisted that EUS-FNA has better tolerability than EBUS-TBNA. In clinical practice, we occasionally encounter patients with severe cough during the EBUS-TBNA [5], which is likely to occur less frequently during EUS-B-FNA. Furthermore, oxygen desaturation in $6 \%$ of the cases during EUS-B-FNA in the current study appears to be less fre- quent than during bronchoscopy [32]. Although a certain experience and skill may be necessary to obtain satisfactory specimens, the EUS-B-FNA procedure does not seem so difficult for pulmonologists who are familiar with handling ultrasound bronchoscopes. Our study indicates that EUS-B-FNA is sensitive enough for use as the sole diagnostic tool in the pathological diagnosis of sarcoidosis. Thus, this procedure, as well as EUS-FNA or EBUS-TBNA, may be the procedure of choice for experienced examiners. Further comparative study with EBUSTBNA or EUS-FNA with the focus on diagnostic accuracy and patient tolerability may be required.

In conclusion, our findings suggest that EUS-B-FNA is feasible, safe and accurate for the diagnosis of stage I/ II sarcoidosis in detecting noncaseating epitheloid cell granulomas. This procedure may be a useful alternative to conventional EUS-FNA or EBUS-TBNA.

\section{Acknowledgments}

This study was supported in part by a Japanese Foundation for Research and Promotion of Endoscopy grant (2010).

\section{Financial Disclosure and Conflicts of Interest}

The authors have no conflicts of interest to disclose.

\section{References}

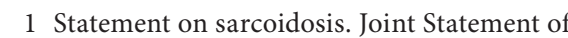
the American Thoracic Society (ATS), the European Respiratory Society (ERS) and the World Association of Sarcoidosis and Other Granulomatous Disorders (WASOG) adopted by the ATS Board of Directors and by the ERS Executive Committee, February 1999. Am J Respir Crit Care Med 1999;160:736755.

- Nakajima T, Yasufuku K, Kurosu K, Takiguchi Y, Fujiwara T, Chiyo M, Shibuya K, Hiroshima K, Nakatani Y, Yoshino I: The role of EBUS-TBNA for the diagnosis of sarcoidosis: comparisons with other bronchoscopic diagnostic modalities. Respir Med 2009;103: 1796-1800.

-3 de Boer S, Milne DG, Zeng I, Wilsher ML: Does CT scanning predict the likelihood of a positive transbronchial biopsy in sarcoidosis? Thorax 2009;64:436-439.
-4 Navani N, Booth HL, Kocjan G, Falzon M, Capitanio A, Brown JM, Porter JC, Janes SM: Combination of endobronchial ultrasound guided transbronchial needle aspiration with standard bronchoscopic techniques for the diagnosis of stage I and stage II pulmonary sarcoidosis. Respirology 2011;16:467-472.

$\checkmark 5$ Oki M, Saka H, Kitagawa C, Kogure Y, Murata N, Ichihara S, Moritani S: Prospective study of endobronchial ultrasound-guided transbronchial needle aspiration of lymph nodes versus transbronchial lung biopsy of lung tissue for diagnosis of sarcoidosis. J Thorac Cardiovasc Surg 2012;143:13241329.

-6 Herth FJ, Rabe KF, Gasparini S, Annema JT: Transbronchial and transoesophageal (ultrasound-guided) needle aspirations for the analysis of mediastinal lesions. Eur Respir J 2006;28:1264-1275.

7 Varela-Lema L, Fernández-Villar A, RuanoRavina A: Effectiveness and safety of endobronchial ultrasound-transbronchial needle aspiration: a systematic review. Eur Respir J 2009;33:1156-1164.
8 Fritscher-Ravens A, Sriram PV, Topalidis T, Hauber HP, Meyer A, Soehendra N, Pforte A: Diagnosing sarcoidosis using endosonography-guided fine-needle aspiration. Chest 2000;118:928-935.

-9 Annema JT, Veseliç M, Rabe KF: Endoscopic ultrasound-guided fine-needle aspiration for the diagnosis of sarcoidosis. Eur Respir J 2005;25:405-409.

10 Iwashita T, Yasuda I, Doi S, Kato T, Sano K, Yasuda S, Nakashima M, Hirose Y, Takaimi T, Moriwaki H: The yield of endoscopic ultrasound-guided fine needle aspiration for histological diagnosis in patients suspected of stage I sarcoidosis. Endoscopy 2008;40: 400-405.

11 von Bartheld MB, Veseliç-Charvat M, Rabe KF, Annema JT: Endoscopic ultrasoundguided fine-needle aspiration for the diagnosis of sarcoidosis. Endoscopy 2010;42: 213-217. 
12 Tournoy KG, Bolly A, Aerts JG, Pierard P, De Pauw R, Leduc D, Leloup A, Pieters T, Slabbynck H, Janssens A, Carron K, Schrevens L, Pat K, De Keukeleire T, Dooms C: The value of endoscopic ultrasound after bronchoscopy to diagnose thoracic sarcoidosis. Eur Respir J 2010;35:1329-1335.

13 Wong M, Yasufuku K, Nakajima T, Herth FJ, Sekine Y, Shibuya K, Iizasa T, Hiroshima K, Lam WK, Fujisawa T: Endobronchial ultrasound: new insight for the diagnosis of sarcoidosis. Eur Respir J 2007;29:1182-1186.

14 Oki M, Saka H, Kitagawa C, Tanaka S, Shimokata T, Kawata Y, Mori K, Kajikawa S, Ichihara S, Moritani S: Real-time endobronchial ultrasound-guided transbronchial needle aspiration is useful for diagnosing sarcoidosis. Respirology 2007;12:863-868.

15 Garwood S, Judson MA, Silvestri G, Hoda R, Fraig M, Doelken P: Endobronchial ultrasound for the diagnosis of pulmonary sarcoidosis. Chest 2007;132:1298-1304.

16 Tremblay A, Stather DR, Maceachern P, Khalil M, Field SK: A randomized controlled trial of standard vs endobronchial ultrasonography-guided transbronchial needle aspiration in patients with suspected sarcoidosis. Chest 2009;136:340-346.

-17 Hwangbo B, Lee HS, Lee GK, Lim KY, Lee SH, Kim HY, Lee JY, Zo JI: Transoesophageal needle aspiration using a convex probe ultrasonic bronchoscope. Respirology 2009; 14: 843-849.
18 Oki M, Saka H, Kitagawa C, Sato S: Bronchoscopic transesophageal ultrasound-guided needle aspiration: an alternative to the conventional transesophageal ultrasound-guided needle aspiration technique. J Thorac Cardiovasc Surg 2010;139:1659-1661.

$\checkmark 19$ Hwangbo B, Lee GK, Lee HS, Lim KY, Lee SH, Kim HY, Lee HS, Kim MS, Lee JM, Nam BH, Zo JI: Transbronchial and transesophageal fine needle aspiration using an ultrasound bronchoscope in mediastinal staging of potentially operable lung cancer. Chest 2010;138:795-802.

20 Herth FJ, Krasnik M, Kahn N, Eberhardt R, Ernst A: Combined endoesophageal-endobronchial ultrasound-guided, fine-needle aspiration of mediastinal lymph nodes through a single bronchoscope in $150 \mathrm{pa}$ tients with suspected lung cancer. Chest 2010;138:790-794.

21 Medford AR, Agrawal S: Single bronchoscope combined endoscopic-endobronchial ultrasound-guided fine-needle aspiration for tuberculous mediastinal nodes. Chest 2010;138:1274.

22 Oki M, Saka H, Kitagawa C: Transesophageal bronchoscopic ultrasound-guided fineneedle aspiration for diagnosis of peripheral lung cancer. Ann Thorac Surg 2011;91:16131616.

23 Oki M, Saka H, Kitagawa C, Kogure Y, Murata N, Ichihara S, Moritani S, Ando M: Randomized study of 21-gauge versus 22-gauge endobronchial ultrasound-guided transbronchial needle aspiration needles for sampling histology specimens. J Bronchol Inter Pulmonol 2011;18:306-310.

24 Mountain CF, Dresler CM: Regional lymph node classification for lung cancer staging. Chest 1997;111:1718-1723.
25 British Thoracic Society Bronchoscopy Guidelines Committee, a Subcommittee of Standards of Care Committee of British Thoracic Society. British Thoracic Society guidelines on diagnostic flexible bronchoscopy. Thorax 2001;56(suppl 1):i1-i21.

26 Tambouret R, Geisinger KR, Powers CN, Khurana KK, Silverman JF, Bardales R, Pitman MB: The clinical application and cost analysis of fine-needle aspiration biopsy in the diagnosis of mass lesions in sarcoidosis. Chest 2000;117:1004-1011.

27 Vilmann P, Jacobsen GK, Henriksen FW, Hancke S: Endoscopic ultrasonography with guided fine needle aspiration biopsy in pancreatic disease. Gastrointest Endosc 1992;38: 172-173.

28 Annema JT, Rabe KF: Why respiratory physicians should learn and implement EUSFNA. Am J Respir Crit Care Med 2007;176: 99.

-29 Cetinkaya E, Yilmaz A, Ozgul A, Gencoglu A, Gunluoglu G: Left atrial mass demonstrated during endobronchial ultrasound session. Respiration 2011;81:57-58.

30 Annema JT, Rabe KF: Endosonography for lung cancer staging: one scope fits all? Chest 2010;138:765-767.

31 Wang KP, Feller-Kopman D, Mehta A, Sharma M, Yarmus L: Endobronchial ultrasound and esophageal ultrasound: just because we can, does not necessarily mean we should. Chest 2011;140:271-272.

32 Jones AM, O'Driscoll R: Do all patients require supplemental oxygen during flexible bronchoscopy? Chest 2000;119:1906-1909. 